\title{
Distinguishing core and antenna fucosylated glycopeptides based on low energy tandem mass spectra
}

\author{
András Ács, ${ }^{1,2)}$ Oliver Ozohanics, ${ }^{13)}$ Károly Vékey, ${ }^{1)}$ László Drahos, ${ }^{1)}$ Lilla Turiák*1)
}

1) MS Proteomics Research Group, Research Centre for Natural Sciences, Hungarian Academy of Sciences, Magyar Tudósok körútja 2., H-1117, Budapest, Hungary

2) Semmelweis University, PhD School of Pharmaceutical Sciences, Üllöi út 26, H-1085, Budapest, Hungary

3) Semmelweis University, Department of Medical Biochemistry, Tüzoltó út 37-47, H-1094, Budapest, Hungary

KEYWORDS: mass spectrometry, glycopeptide, fragmentation, CID, energy-dependent, fucosylation

ABSTRACT: A straightforward approach has been developed to distinguish core and antenna fucosylation in glycopeptides. The method does not require derivatization, and can be easily adapted into a proteomics workflow. The key aspect is to use low collision energy CID (on a QTOF type instrument) when only single step fragmentation processes occur. Low collision energy should show the precursor ion as the largest peak in the spectrum; the survival yield should be ideally over 50\%; and this is obtained at a collision energy ca. $30 \%$ of that typically used for proteomics. In such a case interfering processes like fucose migration or consecutive reactions are minimized. Core and antenna fucosylation can be discriminated using various ion abundance ratios. Low energy CID spectra are very "clean" (no chemical noise), and the ions used for locating the fucose are among the major peaks; making the method well suited for analytical work. Monitoring the change in the proportion of core and antenna fucosylation at the same glycosylation site is also feasible. 


\section{INTRODUCTION}

Glycoprotein analysis has gained importance in the recent decade, both with respect to glycoproteomics ${ }^{1-4}$ and to therapeutically used monoclonal antibodies (MABs) ${ }^{5-7}$ In most cases a peptide/glycopeptide mixture is analyzed by HPLC-MS/MS. This approach has many advantages, but its main disadvantage is that glycopeptide MS/MS analysis yields relatively little information on the oligosaccharide structure. One often requested information relates to fucosylation, in particular whether core or antenna fucosylation is present.

Glycoprotein fucosylation is often used as a biomarker for various diseases. ${ }^{8,9}$ Fucosylation takes place frequently on the $\mathrm{N}$-acetylhexosamine units of $\mathrm{N}$-glycans, mostly on the core (connected directly to the peptide chain), occasionally on the antenna. Core fucosylation of the $\mathrm{N}$ acetylhexosamine residue occurs by $\alpha 1-6$ linkage, ${ }^{10}$ while antenna fucosylation by $\alpha 1-3$ linkage $\mathrm{e}^{10}$ (occasionally, $\alpha 1-4$ linkage may also occur). ${ }^{10}$ Fucosylation may also be present on galactose residues of polylactosamine extensions of $\mathrm{N}$-glycans giving rise to $\mathrm{A} / \mathrm{B} / \mathrm{H}$ blood group antigens. ${ }^{11}$ Distinguishing core and antenna fucosylation is often desired but is not trivial. The main problem, that fucose is known to migrate along the oligosaccharide chain during mass spectrometry analysis. ${ }^{12,13}$ Fucose migration, to some degree, may compromise evaluation of stepped collision energy experiments as well, which were recently shown to be useful for glycopeptide analysis. ${ }^{14}$ In order to discriminate core versus antenna fucosylation a procainamide hydrochloride labeling method was recently described and validated on released N-glycans of standard glycoproteins. ${ }^{15}$ Tajiri et. al ${ }^{16}$ has studied energy dependent MS/MS fragmentation of fucosylated glycopeptides; establishing that fucose loss does not compromise determination of the degree of fucosylation; neither in electrospray, nor in MALDI ionization as long as the collisional excitation was kept low. Under such conditions MS ion abundances could be used to estimate fucosylation levels.

Here we will show that analysis of core and antenna fucosylation on $\mathrm{N}$-acetylhexosamine residues is feasible to perform based on low energy MS/MS analysis of glycopeptides. We also show that fucose migration, at least at low collision energy and on a quadrupole-type instrument, occurs only to a minor extent. We did not study galactose fucosylation (on $\mathrm{A} / \mathrm{B} / \mathrm{H}$ blood group antigens), but the present method might be extended to those as well in the future. We have selected two examples. In the case of prostate specific antigen (PSA) core fucosylation occurs (NKSVILLGR 
peptide using Arg-C digestion). ${ }^{17}$ In the case of alpha-1 acid glycoprotein (AGP) antenna fucosylation (at position 56, a tryptic glycopeptide with NEEYNK sequence) is present. ${ }^{15}$

\section{EXPERIMENTAL SECTION}

Chemicals and reagents

The following standards and enzymes were purchased from Sigma-Aldrich (Sigma-Aldrich, St. Louis, MO, USA): alpha-1 acid glycoprotein (AGP) standard, LC-MS grade solvents. Mass spectrometry grade trypsin and sequencing grade Arg-C were obtained from Promega (Promega Corporation, Madison, WI, USA). RapiGest SF (lyophilized sodium-3-[(2-methyl-2-undecyl-1,3dioxolan-4-yl)-methoxyl]-1-propane-sulfonate) was purchased from Waters (Milford, MA, USA), BCR-613 Prostate specific antigen (PSA) reference material standard from European Commission, Joint Research Centre (Geel, Belgium).

Enzymatic digestion

$1 \mathrm{nmol}$ AGP was digested in solution using LysC-trypsin (1:100 ratio, 1 hour) and trypsin (1:10 ratio, 2 hours) enzymes as previously described. ${ }^{18} 250$ pmol PSA was digested using $\operatorname{ArgC}$ (1: 25 ratio) with minor modifications to the above mentioned protocol: Following incubation with iodoacetamide, dithiothreitol and $\mathrm{CaCl}_{2}$ were added to enhance $\mathrm{Arg}-\mathrm{C}$ enzyme activity.

Nano LC-MS(MS)

For the liquid chromatography-mass spectrometry measurements a Maxis II ETD Q-TOF (Bruker Daltonics, Bremen, Germany) equipped with a CaptiveSpray nanoBooster ionsource coupled to an Ultimate 3000 nanoRSLC system (Dionex, Sunnyvale, CA, USA) was used. Samples were dissolved in 2\% AcN, 0,1\% FA and injected onto an Acclaim PepMap100 C-18 trap column (100 $\mu \mathrm{m}$ x $20 \mathrm{~mm}$, Thermo Scientific, Sunnyvale, CA, USA) for sample desalting. Peptides were separated on an ACQUITY UPLC M-Class Peptide BEH C18 column (130 ̊, 1,7 $\mu \mathrm{m}, 75 \mu \mathrm{m} x$ $250 \mathrm{~mm}$, Waters, Milford, MA, USA) at $48{ }^{\circ} \mathrm{C}$ applying a flow rate of $300 \mathrm{nl} / \mathrm{min}$ using gradient elution (4\% B from 0 to $11 \mathrm{~min}$, followed by a $60 \mathrm{~min}$ or $90 \mathrm{~min}$ gradient to $50 \% \mathrm{~B}$ ).

Glycosylation analysis - instrument settings 
The ion transfer tune parameters were set as follows: pre-pulse storage $10 \mu \mathrm{s}$, collision cell collision energy 7 eV, quadrupole ion energy 4 eV, Funnel 1 RF 400 Vpp, Multipole RF 800 Vpp. The collision RF was set to $800 \mathrm{Vpp}$ and the ion transfer time was $140 \mu \mathrm{s}$. The MS spectra were recorded over the mass range of $m / z, 150-3000$ at $5 \mathrm{~Hz}$. The CID was performed on selected triply charged glycopeptide precursors using an inclusion list. The CID was performed at $4 \mathrm{~Hz}$ for abundant precursors and at $1 \mathrm{~Hz}$ for low abundant ones. MS/MS experiments were performed at various collision energies in the 10.9-55 V range. First the "standard" collision energy for precursor signals was determined following the manufacturer's recommendations for peptides based on the isolation $\mathrm{m} / \mathrm{z}$, isolation mass range width and charge state of the ion. In the energy dependent measurements, the collision energy was set to a given percentage of this energy (in the $30 \%-150 \%$ range). Following each run raw data were recalibrated using the Compass DataAnalysis software 4.3 (Bruker Daltonics, Bremen, Germany).

Data evaluation

MS/MS scans for each glycopeptide were summarized at the different energies. Intensities of each product ion were determined; numerical values discussed in the paper always relate to the highest isotope contribution of the selected ion. Breakdown curves were normalized to the maximum abundance of each ion as a function of collision voltage.

\section{RESULTS AND DISCUSSION}

a1-6 core fucosylation (PSA derived NKSVILLGR -N4H5S2F glycoform)

The energy-dependent MS/MS spectra of the triply protonated PSA-BiS2F glycoform (biantennary, fully sialylated, $\alpha 1-6$ core fucosylated, with sugar composition of N4H5S2F) are shown in Fig. 1 (N: N-acetylhexosamine, H: hexose, S: sialic acid, F: fucose). Predominant low energy product ions (Fig. 1A and 1B) are due to cleavages between the sugar residues, leading to singly charged oxonium ions $\left(\mathrm{B}^{+}\right.$ion series, according to the nomenclature of Domon-Costello. ${ }^{19}$ The same reaction also leads to a doubly charged product $\left(\mathrm{M}^{3+} \gg>\mathrm{B}^{+}+[\mathrm{M}-\mathrm{B}]^{2+}\right)$ in a charge separation process..$^{20-22}$ The most abundant process is due to cleavage between the oligosaccharide core and the antenna ( $\mathrm{NHS}^{+}$and $[\mathrm{M}-\mathrm{NHS}]^{2+}$ ), as typically observed in glycopeptides. ${ }^{16,20,23,24}$ The other abundant low energy processes are due to analogous cleavages forming $\mathrm{S}^{+},[\mathrm{M}-\mathrm{S}]^{2+}, \mathrm{HNHS}^{+}$ 
and $[\mathrm{M}-\mathrm{HNHS}]^{2+}$ ions. To simplify discussion we will use $\mathrm{M}^{3+}$ to indicate the triply protonated molecule throughout the paper.

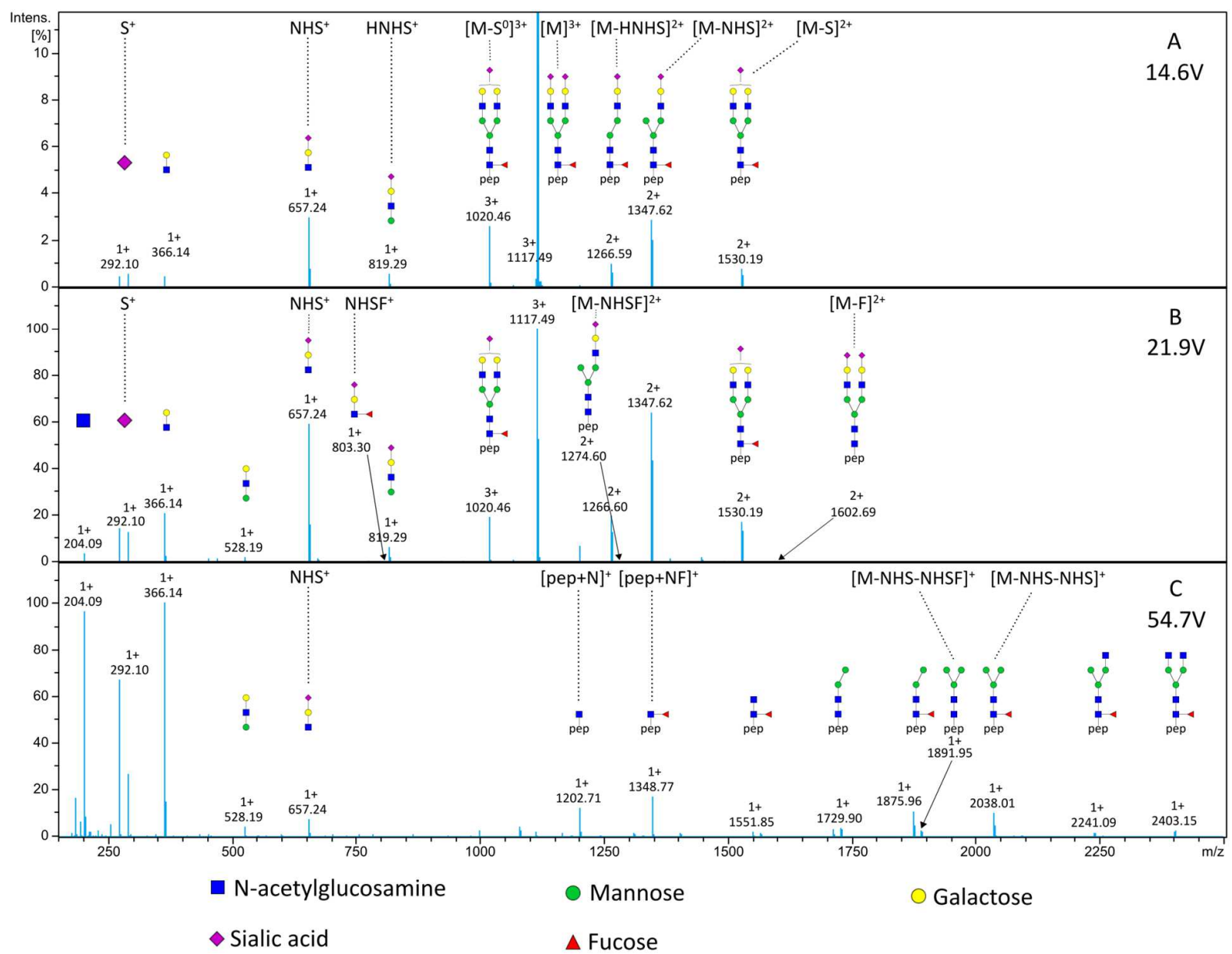

Figure 1. MS/MS spectra of PSA derived NKSVILLGR -N4H5S2F glycopeptide $(\mathrm{m} / \mathrm{z}, 1117.492)$ at $14.6 \mathrm{~V}$ (A) (40\% of the "standard" collision energy), $21.9 \mathrm{~V}$ (B) (60\% of the "standard" collision energy) and 54.7 V (C) (150\% of the "standard" collision energy). Spectra of Fig. 1A was zoomed in on the y-axis to $12 \%$ relative intensity to allow visibility of low abundance product ions. $(\mathrm{N}: \mathrm{N}-$ acetylhexosamine, H: hexose, S: sialic acid, F: fucose).

At low intensity a different process is also observed, leading to the loss of a neutral sialic acid residue (291 Da). This is a Y type ion formation (analogous to that of a B ion, but with hydrogen rearrangement), the product ion having the same charge as the precursor (i.e. triply charged in the present case, $\left.\left[\mathrm{M}-\mathrm{S}^{0}\right]^{3+}\right)$. Note, that the abundance of this process is lower than that of the $\mathrm{B}^{+}$and $[\mathrm{M}-\mathrm{B}]^{2+}$ ion formation. Beside the loss of a neutral sialic acid, loss of a neutral fucose residue is 
also observed (146 Da loss); but its abundance is very low, less than 10\% that of the neutral sialic acid loss. No trace of fucose B ion formation is observed; the best indication is the lack of a doubly charged $[\mathrm{M}-\mathrm{F}]^{2+}$ ion (less than $0.1 \%$ that of $[\mathrm{M}-\mathrm{NHS}]^{2+}$ ). These observations suggest that the core fucose ( $\alpha 1-6$ linkage) is strongly bound to the sugar backbone.

At higher energy (Fig. 1C) two-step and multi-step processes start to appear. These are due to combination of the processes described above. Possibly the most characteristic such process is cleavage of both antenna, like $[\mathrm{M}-\mathrm{NHS}]^{2+}>>\mathrm{NHS}^{+}+[\mathrm{M}-\mathrm{NHS}-\mathrm{NHS}]^{+}$, which is a sequential B ion formation. Neutral monosaccharide residue losses also occur; eventually forming the [peptide $+\mathrm{N}]^{+}$and [peptide $\left.+\mathrm{NF}\right]^{+}$ions, often used by software to identify the peptide mass.

Based on both low and high energy spectra (Fig. 1) it is straightforward to assign fucosylation to the core: At low energy it is indicated by the lack of $\mathrm{NHSF}^{+}$and $[\mathrm{M}-\mathrm{NHSF}]^{2+}$ ions (which may be present in traces only, while the $\mathrm{NHS}^{+}$and $[\mathrm{M}-\mathrm{NHS}]^{2+}$ ion pair is abundant). At high energy the two-step formation of the [M-NHS-NHS $]^{+}$ion also indicates core fucosylation, although the presence of [M-NHS-NHSF $]^{+}$ion may suggest the presence of antenna fucosylation. However, the latter ion may also be explained by fucose migration, ${ }^{12,13}$ or by a three-step sequential process $\left[\mathrm{M}^{3+}-\mathrm{NHS}^{+}-\mathrm{NHS}^{+}-\mathrm{F}^{0}\right]^{+}$.

A more detailed picture of assigning fucose to the core or the antenna may be established by studying energy dependence. The "low" and "high" collision energies, as used above, are relative terms. Glycopeptides fragment more easily (i.e. require less activation energy) than peptides. ${ }^{23,25}$ One often used measure for the collision energy is the survival yield, ${ }^{26}$ i.e. the percentage of the non-fragmented molecular species compared to the sum of all ion abundances. In the present case "low energy" indicates the range of collision energies, when the survival yield is at least $10 \%$ (i.e. the molecular ion is among the abundant ions in the spectrum), while "high energy" indicates the collision energy range, when the molecular ion is practically absent (the survival yield is less than $1 \%)$. It is also worth comparing the collision energies used to that of the "standard" collision energy suggested by the manufacturer (which changes with the mass and charge of the precursor ion) for peptides. In the present case fragmentation starts at $10.9 \mathrm{~V}$ collision voltage $(30 \%$ of the standard collision energy), 50\% survival yield is obtained at ca. $19.3 \mathrm{~V}(53 \%), 10 \%$ survival yield 
is at about $26.6 \mathrm{~V} \mathrm{(73 \% ),} \mathrm{and} \mathrm{the} \mathrm{molecular} \mathrm{ion} \mathrm{disappears} \mathrm{from} \mathrm{the} \mathrm{spectra} \mathrm{at} \mathrm{about} 29.2 \mathrm{~V}(80 \%$ of the "standard" collision energy).

Energy dependence of major processes can be best studied by breakdown diagrams, i.e. ion abundances as a function of collision energy. For the triply protonated PSA-BiS2F ion these are shown in Fig. 2; the collision energy is indicated as a \% of the "standard" collision energy. To offer easier comparison of various processes, the intensity of each ion is normalized separately (i.e. reaches $100 \%$ at the collision energy, where its relative intensity is the highest). Fig. 2A shows the breakdown curve of $\mathrm{M}^{3+}$; that of the main charge separation process $\left(\mathrm{NHS}^{+}\right.$and $[\mathrm{M}-\mathrm{NHS}]^{2+}$ ), and a two-step process [M-NHS-NHS $]^{+}$. This indicates that at low energy the curves of $\mathrm{NHS}^{+}$and $[\mathrm{M}-\mathrm{NHS}]^{2+}$ are overlapping (as they should). The single-step and two-step processes are well separated. $\mathrm{NHS}^{+}$is formed in both the single-step and two-step process, so that above $80 \%$ collision energy the breakdown curve of $\mathrm{NHS}^{+}$has a high energy tail. Fig. 2B show the breakdown curves of neutral monosaccharide (sialic acid and fucose) losses. This indicates that although the corresponding ions are of relatively low abundance, their formation requires lower activation energy, than that of $\mathrm{NHS}^{+}$or $[\mathrm{M}-\mathrm{NHS}]^{2+}$. Fig. 2B also shows breakdown curve of the $[\mathrm{M}-\mathrm{NHSF}]^{2+}$, and as a comparison, that of $[\mathrm{M}-\mathrm{NHS}]^{2+}$, which was also in Fig. 2A. Note that the curve for [M$\mathrm{NHSF}]^{2+}$ starts at a significantly higher collision energy than that of $[\mathrm{M}-\mathrm{NHS}]^{2+}$; which suggests that the $[\mathrm{M}-\mathrm{NHSF}]^{2+}$ ion is formed in a two-step process: $\mathrm{M}^{3+}-\mathrm{F}^{0}-\mathrm{NHS}^{+}$. Note, if there were antenna fucose present, the same ion would be formed in a single step process, but that would require lower activation energy; and the breakdown curve would be practically overlapping with that of $[\mathrm{M}-\mathrm{NHS}]^{2+}$. Such a case is discussed below (Fig.5), where (in the case of antenna fucosylation) single-step $\mathrm{NHS}^{+}$and $\mathrm{NHSF}^{+}$losses show practically overlapping breakdown curves. 


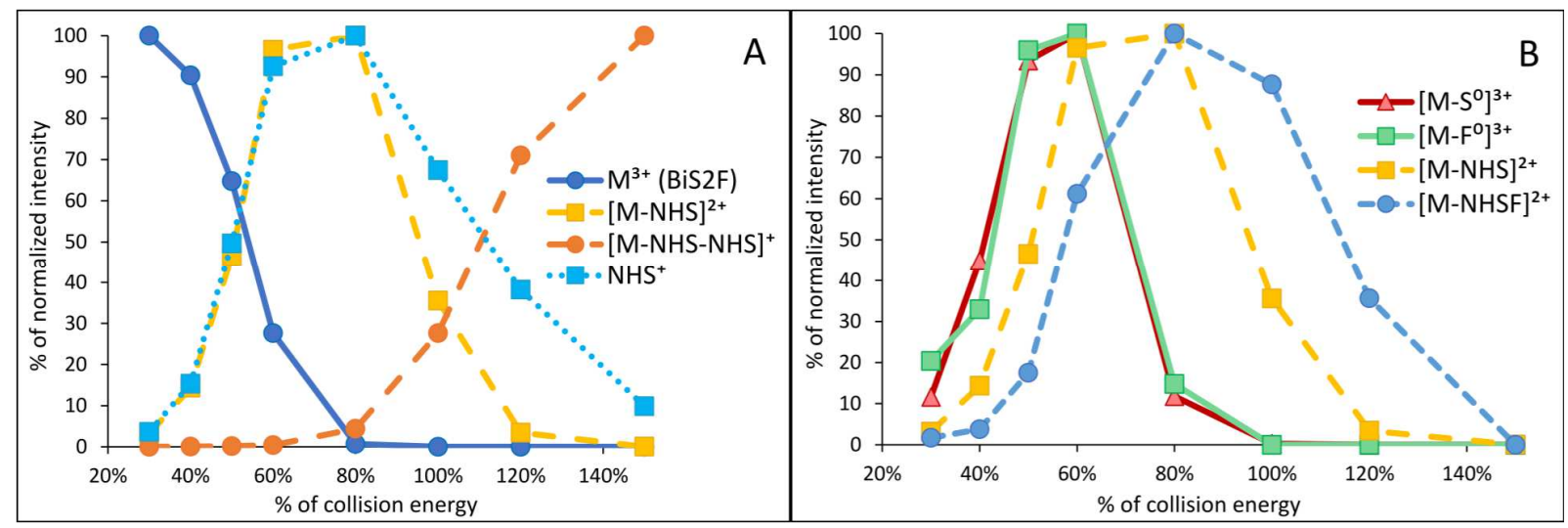

Figure 2 Breakdown curves of PSA derived NKSVILLGR -N4H5S2F glycopeptide $(\mathrm{m} / \mathrm{z}$ 1117.492 ) in the $30-150 \%$ "standard" collision energy range (x-axis); normalized to the maximum abundance of each ion as a function of collision voltage for product ions. (A) $\mathrm{M}^{3+},[\mathrm{M}-\mathrm{NHS}]^{2+}$, $[\mathrm{M}-\mathrm{NHS}-\mathrm{NHS}]^{2+}$ and $\mathrm{NHS}^{+}$are shown. (B) $\left[\mathrm{M}-\mathrm{S}^{0}\right]^{3+},\left[\mathrm{M}-\mathrm{F}^{0}\right]^{3+},[\mathrm{M}-\mathrm{NHS}]^{2+}$ and $[\mathrm{M}-\mathrm{NHSF}]^{2+}$ are shown.

As discussed above, assigning core and antenna fucosylation may be based on various peaks in the spectra. Using peak ratios can give a quantitative estimate of the proportion of core and antenna fucosylation in a sample. At low energy (when single step processes dominate) Eq. 1 gives an estimate of antenna fucosylation:

$$
F_{1}=n * \frac{[M-N H S F]^{2+}}{[M-N H S]^{2+}+[M-N H S F]^{2+}} \quad \text { Eq. } 1
$$

Here the ions represent the respective ion abundances, while $n$ is the number of antennae. In the present example $n=2$, the $2 \mathrm{x}$ multiplication factor compensates for the fact that there are two antennae, and only one can be fucosylated. This equation rests on the assumption that probability of antenna cleavage does not depend on the presence of fucose on the antenna; and that subsequent fragmentation of $[\mathrm{M}-\mathrm{NHS}]^{2+}$ and $[\mathrm{M}-\mathrm{NHSF}]^{2+}$ are equally likely; and that there is no contribution of two-step processes. A second estimate is based on the oxonium ions:

$$
\begin{array}{ll}
F_{2}=n * \frac{N H S F^{+}}{N H S^{+}+N H S F^{+}} & \text {Eq. } 2
\end{array}
$$

This is likely to be a strong underestimate of antenna fucosylation (or fucose migration), as NHSF ${ }^{+}$ is known to fragment much more easily, than $\mathrm{NHS}^{+}$. 
A third estimate is based on two-step processes (observable at medium or high collision energy), when both antennae are lost:

$$
F_{3}=\frac{[M-N H S-N H S F]^{2+}}{[M-N H S-N H S]^{2+}+[M-N H S-N H S F]^{2+}} \quad \text { Eq. } 3
$$

We have measured the values of $F_{1}, F_{2}$ and $F_{3}$ regarding the PSA-BiS2F glycoform as a function of collision energy, and these are shown in Fig. 3. The $F_{2}$ is likely to be a significant underestimation, due to the easy fragmentation of $\mathrm{NHSF}^{+}$. This value, however, is direct proof of fucose migration; and gives a lower limit for fucose migration. $F_{1}$ and $F_{3}$, on the other hand are due to a combination of both fucose migration and the consequence of multi-step processes. Our estimate is that multi-step processes have a larger contribution to this, than fucose migration. Both $F_{1}$ and $F_{3}$ show a fast increase with collision energy. This is due to an increasing amount of fucose migration from the core to the antenna, and by the increasing role of multi-step processes at higher collision energies (i.e. at higher internal energy). It is very pleasing that $F_{1}$ and $F_{3}$ yield approximately the same result in the middle energy range (60-100\% of the "standard" collision energy), when both single and multiple step processes can be observed. The $F_{2}$ estimate does not change significantly with energy - likely because increased amount of fucose migration, and increased fragmentation of $\mathrm{NHSF}^{+}$compensate each other. 


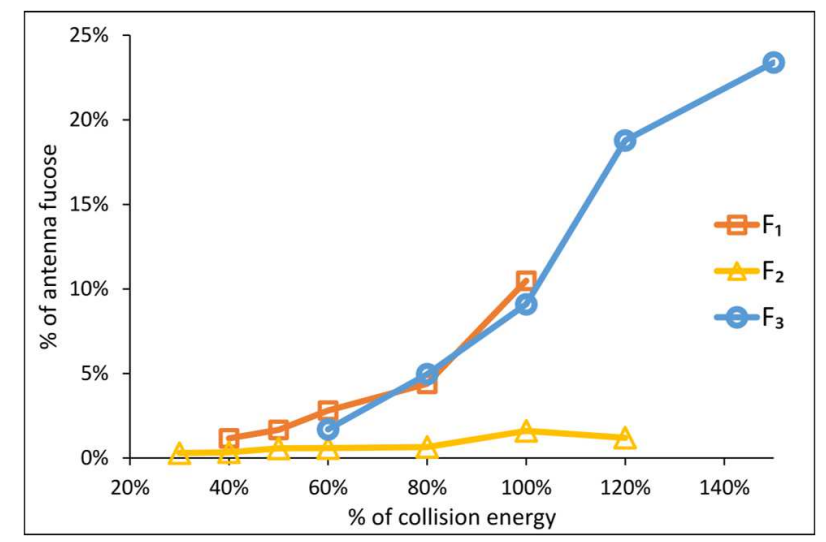

Figure 3. The $\%$ of antenna fucosylation estimated by ion abundance ratios according to Equations 1, 2 and 3, as a function of "standard" collision energy.

The results show that at very low energy there is only little interference due to fucose migration from the core to the antenna, and also little interference due to multi-step processes. The best estimate of antenna fucosylation may be based on Eq. 1 at low energy (40-50\% that of the "standard" collision energy, when the survival yield is over 40\%). Under such conditions the threshold level of antenna fucosylation is $2-3 \%$ (i.e. which is due to fucose migration and multistep processes). If the $F_{1}$ parameter is significantly above this threshold, that would indicate the presence of antenna fucosylation. The degree of antenna fucosylation might be determined at higher energies as well, although less accurately: Up to collision energies when the molecular ion is easily observable (has at least $1 \%$ intensity, in the present case ca. $80 \%$ that of the "standard" collision energy) the error due to fucose migration and multi-step processes is still fairly low, ca. $5 \%$. The presence of core fucosylation may be identified even at higher energies when both the molecular ion and doubly charged primary product ions are absent from the spectra (Fig. 1C, 150\% "standard" collision energy), based on the presence of the [peptide+NF] ${ }^{+}$ion. However, the contribution of antenna fucosylation will not be possible to exclude at high energy.

Indication of core fucosylation may be determined based on doubly or quadruply protonated precursors as well. However, in the case of doubly protonated precursors oxonium ions require larger activation energy to fragment, ${ }^{20}$ so that contribution of fucose migration and neutral fucose residue loss will be larger. In the case of quadruply protonated precursors multi-step processes will require less energy, so the contribution of multi-stage processes will be larger. Both effects will 
increase the estimate of antenna fucosylation based on Equation 1 and 3; so the use of triply protonated precursors seems optimal for fucose localization.

ג1-3 antenna fucosylation (AGP derived NEEYNK -N4H5S2F glycoform)

Antenna fucosylation is found in alpha 1-acid glycoprotein, where the triply protonated NEEYNKBiS2F glycoform is studied. According to the literature, ${ }^{15,27}$ AGP contains only antenna fucosylation with $\alpha 1-3$ linkage (antenna fucosylation may also involve 1-4 linkage as well, but this was not studied). MS/MS spectra are shown in Fig. 4 at various collision energy values.

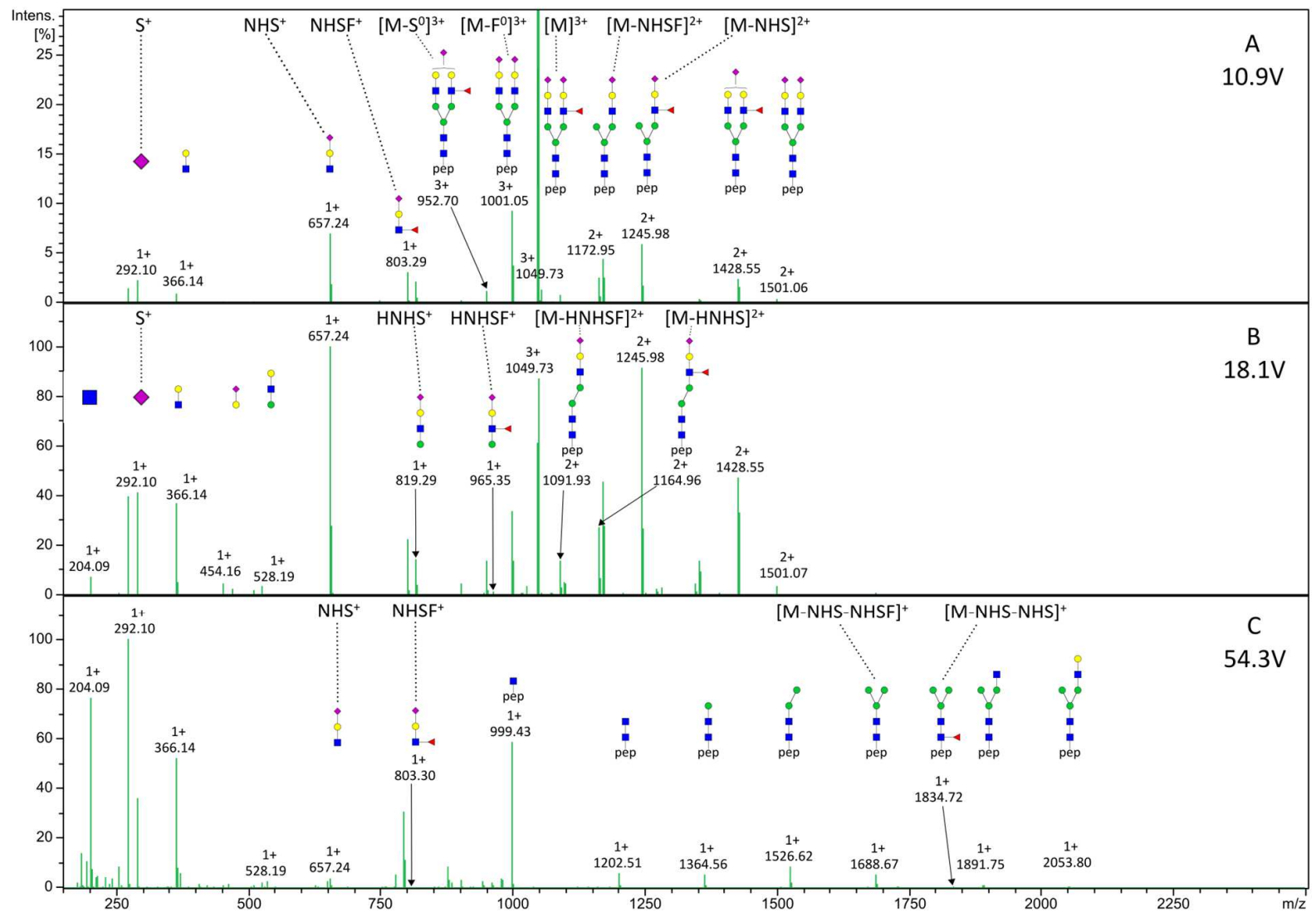

Figure 4. MS/MS spectra of AGP derived NEEYNK -N4H5S2F glycopeptide $(\mathrm{m} / z$ 1049.7258) at $10.9 \mathrm{~V}$ (A) (30\% of the "standard" collision energy), $18.1 \mathrm{~V}$ (B) (50\% of the "standard" collision energy) and $54.3 \mathrm{~V}(\mathrm{C})$ (150\% of the "standard" collision energy). Spectra of Fig. 4A was zoomed in on the y-axis to $25 \%$ relative intensity to allow visibility of low abundance product ions. 
At low energy (30\% of the standard collision energy, 10.9 V, Fig. 4A) neutral fucose loss is among the most abundant processes; the peak intensity is nearly 10 times larger, than that of the analogous neutral sialic acid loss. This indicates that the antenna fucose (at least with $\alpha 1-3$ linkage) is less strongly bound, than the core fucose ( $\alpha 1-6$ linkage). Therefore both fucose migration and the contribution of multi-step processes are likely to be more significant, than in the case of core fucosylation. As before, at low energy, the antenna-derived $\mathrm{B}$ ions $\left(\mathrm{S}^{+}, \mathrm{NHS}^{+}, \mathrm{NHSF}^{+}, \mathrm{HNHS}^{+}\right.$ and $\mathrm{HNHSF}^{+}$), and their doubly charged complement ions are among the most abundant product ions. One of the antennae is fucosylated, the other is non-fucosylated, so that both fucosecontaining and fucose non-containing ions are abundant. Antenna fucosylation can be easily identified e.g. by the presence of $\mathrm{NHSF}^{+}\left(\mathrm{m} / \mathrm{z}\right.$ 803) and $[\mathrm{M}-\mathrm{NHSF}]^{2+}$ ions. All of these ions are abundant at low energy, so they are analytically very useful. However, the presence of these ions do not eliminate the possibility that both antenna and core fucosylation might be present.

Fig. 5 shows the breakdown curves for a few important ions: That of the triply protonated molecule $\left(\mathrm{M}^{3+}\right)$, the main primary product ion $[\mathrm{M}-\mathrm{NHS}]^{2+}$, and the peak formed by neutral sialic acid residue loss $\left[\mathrm{M}-\mathrm{S}^{0}\right]^{3+}$, are analogous to those shown in Fig. 2. Just like in the case of core fucosylation, neutral sialic acid residue loss requires less activation energy than $[\mathrm{M}-\mathrm{NHS}]^{2+}$, even though the former is a small peak. The other neutral loss, that of the fucose residue $\left(\left[\mathrm{M}-\mathrm{F}^{0}\right]^{3+}\right)$ is shifted to significantly lower energy than that of $\left[\mathrm{M}-\mathrm{S}^{0}\right]^{3+}$, indicating that the former is a very low energy process. This explains its high intensity at low energy; and suggests that all fucose-containing ions will easily loose a neutral fucose, thus complicating data evaluation. The last breakdown curve in Fig 5. is that of $[\mathrm{M}-\mathrm{NHSF}]^{2+}$. It is similar, but somewhat broader than that of $[\mathrm{M}-\mathrm{NHS}]^{2+}$. This illustrates, that both the single step $\left(\mathrm{M}^{3+}-\mathrm{NHSF}^{+}\right)$and a two-step process, $\mathrm{M}^{3+}-\mathrm{F}^{0}-\mathrm{NHS}^{+}$are likely to contribute significantly to the formation of $[\mathrm{M}-\mathrm{NHSF}]^{2+}$ ion. 


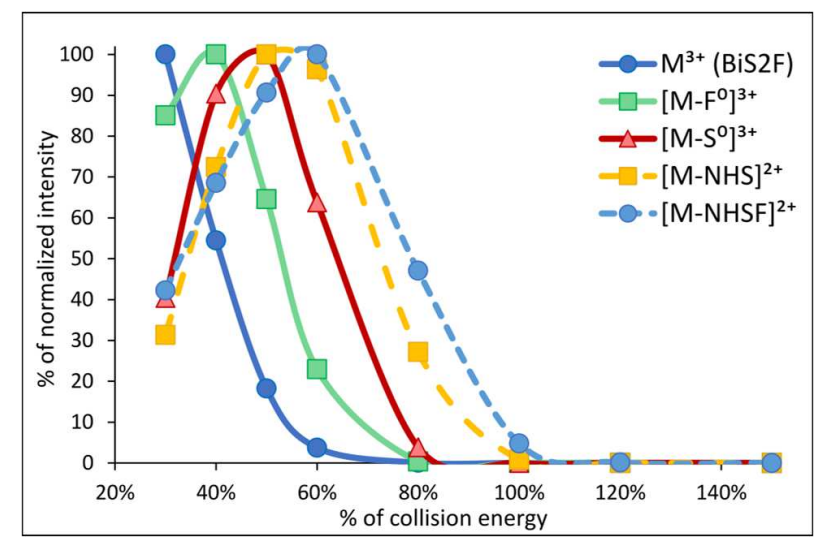

Figure 5. Breakdown curves of AGP derived NEEYNK -N4H5S2F glycopeptide $(\mathrm{m} / \mathrm{z}$ 1049.7258) in the $30-150 \%$ "standard" collision energy range (x-axis); normalized to the maximum abundance of each ion as a function of collision voltage for product ions. $\mathrm{M}^{3+},\left[\mathrm{M}-\mathrm{F}^{0}\right]^{3+},\left[\mathrm{M}-\mathrm{S}^{0}\right]^{3+}[\mathrm{M}-$ $\mathrm{NHS}]^{2+}$ and $[\mathrm{M}-\mathrm{NHSF}]^{2+}$ are shown.

At medium and high energy $(18.1 \mathrm{~V}, 50 \%$ of the "standard" collision energy and $54.3 \mathrm{~V}, 150 \%$ of the "standard" collision energy, Fig 4B and 4C) the spectra show predominantly two-stage and multi-stage fragmentations, leading to singly charged product ions. Among these the loss of both antennae contain the most important structural information, and these are among the most abundant high mass ions. When antenna fucosylation is present, this process is the sequential loss of $\mathrm{NHS}^{+}$and $\mathrm{NHSF}^{+}$; forming the $[\mathrm{M}-\mathrm{NHS}-\mathrm{NHSF}]^{+}$ion. This is indeed a large peak in the high energy spectrum. Whether there is core fucosylation, fucose migration from the antenna to the core, or if neutral fucose residue loss also takes place, then the [M-NHS-NHS] ${ }^{+}$ion will also be present. The spectrum indeed shows this ion (Fig. 4.).

The fraction of antenna fucosylation can be estimated by Equations 1-3, as discussed above, and are shown in Fig. 6. This shows that (in contrast to core fucosylation) the various estimated values $\left(F_{1}, F_{2}\right.$ and $\left.F_{3}\right)$ are significantly different and show a strong energy dependence even at very low collision energy. The value based on $[\mathrm{M}-\mathrm{NHSF}]^{2+}\left(\mathrm{F}_{1}\right)$ is underestimated, partly due to fucose migration (from the antenna to the core) and partly due to the loss of neutral fucose: Either by the $\mathrm{M}^{3+}-\mathrm{F}^{0}-\mathrm{NHS}^{+}$, or by the $\mathrm{M}^{3+}-\mathrm{NHS}^{+}-\mathrm{F}^{0}$ process. At low energy (30\% that of the "standard" collision energy, $70 \%$ survival yield) $86 \%$ antenna fucose is suggested using Equation 1 . As AGP 
is known to contain only antenna fucosylation, the results suggest that even at low energy, interfering processes have a significant effect - much larger, than in the case of core fucosylation. Simply stated, even at low energy there is $14 \%$ fucose migration from the antenna. The effect of fucose migration and neutral fucose loss increases very fast with collision energy: $31 \%$ at $40 \%$ collision energy (40\% survival yield).

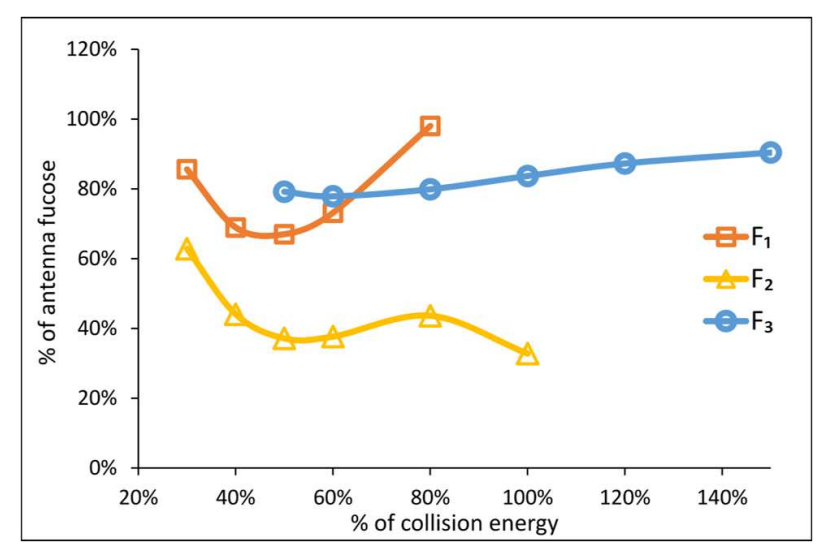

Figure 6. The \% of antenna fucosylation estimated by ion abundance ratios according to Equations 1,2 and 3, as a function of "standard" collision energy.

Estimates based on Equation 2 (i.e. oxonium ions, like $\mathrm{NHSF}^{+}, \mathrm{m} / z$ 803) are even worse, due to the easy fragmentation of $\mathrm{NHSF}^{+}$. At $30 \%$ that of the standard collision energy $\mathrm{F}_{2}$ indicates only $37 \%$ fucose migration, and this estimate further decreases with collision energy. The degree of fucose migration (including the effect of interfering fragmentations) is ca. 20\% based on Equation 3; but this estimate changes only little with collision energy.

\section{CONCLUSIONS}

Identification of the fucosylation site is important for various biological processes. Here we have shown that it is possible to determine the position of fucosylation based on low energy CID spectra of glycopeptides. Analysis of the fucosylation site is thought to be compromised by facile fucose migration. Here we have shown that fucose migration indeed happens, but decreases fast with lowering the collision energy. Besides fucose migration, sequential processes involving neutral fucose residue loss interfere with determining the site of fucosylation; but the importance of such processes also decreases with collision energy. Using low collision energy CID, when the 
precursor ion is the largest peak in the spectrum (i.e. over 50\% survival yield), the effect of fucose migration and other interfering processes can be minimized. We suggest using triply protonated precursors as interfering processes are likely to have least importance in this case. The present study used QTOF type tandem mass spectrometer: fragmentation characteristics and the degree of fucose migration might be different on ion trap instruments.

The core fucose ( $\alpha 1-6$ linkage) is strongly bound to the sugar core; in contrast, the antenna fucose ( $\alpha 1-3$ linkage) is very loosely bound and requires very low activation energy to fragment and migrate. If there is only core or only antenna fucosylation present, these can be discriminated easily in low energy CID spectra, in spite of fucose migration, based on ion abundance ratios like $\mathrm{NHSF}^{+} / \mathrm{NHS}^{+}$or $[\mathrm{M}-\mathrm{NHSF}]^{2+} /[\mathrm{M}-\mathrm{NHS}]^{2+}$. Antenna and core fucosylation may also be based at higher collision energy sequential reaction products like [M-NHS-NHSF] $]^{+}\left[[\mathrm{M}-\mathrm{NHS}-\mathrm{NHS}]^{+}\right.$or $[\text { peptide }+\mathrm{NF}]^{+} /[\text {peptide }+\mathrm{N}]^{+}$. When both core and antenna fucosylation may be present we suggest using Eq. 1 (the ion ratio $\mathrm{F}_{1}=[\mathrm{M}-\mathrm{NHSF}]^{2+} /\left([\mathrm{M}-\mathrm{NHS}]^{2+}+[\mathrm{M}-\mathrm{NHSF}]^{2+}\right)$ to estimate the amount of antenna fucosylation. Note however, that in the case of core fucosylation there will be ca. $2 \%$; in the case of antenna fucosylation $14 \%$ interference due to fucose migration and consecutive processes.

For analytical work; if reference compounds are available, quantitation would be easy either based on Eq. 1; or on the relative abundance of some major characteristic peaks indicated above. When standards are not available, Eq. 1 or changes in the abundance ratios can be used to determine changes in the ratio of antenna and core fucosylation among various samples. Note, that using low energy CID is advantageous not only to minimize fucose migration, but also because the spectra are very "clean" (no chemical noise), and the peaks of interest are among the most abundant peaks in the spectra.

\section{Corresponding Author}

* Lilla Turiák: turiak.lilla@ttk.mta.hu,(+36-1) 3826516

\section{Acknowledgements}

LT, LD and KV are grateful for funding from the National Research Development and Innovation Office (NKFIH PD-121187, NKFIH K-109006 and NKFIH K-119459). LT acknowledges support 
from the János Bolyai Research Scholarship of the Hungarian Academy of Sciences. The research was also supported by the MTA $\square$ MedInProt Programme of the Hungarian Academy of Sciences.

\section{REFERENCES}

(1) Rudd, P.; Karlsson, N. G.; Khoo, K. H.; Packer, N. H. In Essentials of Glycobiology, Varki, A.; Cummings, R. D.; Esko, J. D.; Stanley, P.; Hart, G. W.; Aebi, M.; Darvill, A. G.; Kinoshita, T.; Packer, N. H.; Prestegard, J. H.; Schnaar, R. L.; Seeberger, P. H., Eds.; 2015-2017 by The Consortium of Glycobiology Editors, La Jolla, California: Cold Spring Harbor NY, 2015, pp 653666.

(2) Yang, Y.; Franc, V.; Heck, A. J. R. Glycoproteomics: A Balance between High-Throughput and In-Depth Analysis Trends Biotechnol 2017, 35, 598-609.

(3) Banazadeh, A.; Veillon, L.; Wooding, K. M.; Zabet-Moghaddam, M.; Mechref, Y. Recent advances in mass spectrometric analysis of glycoproteins Electrophoresis 2017, 38, 162-189.

(4) Alley, W. R., Jr.; Mann, B. F.; Novotny, M. V. High-sensitivity analytical approaches for the structural characterization of glycoproteins Chem Rev 2013, 113, 2668-2732.

(5) Plomp, R.; Bondt, A.; de Haan, N.; Rombouts, Y.; Wuhrer, M. Recent Advances in Clinical Glycoproteomics of Immunoglobulins (Igs) Mol Cell Proteomics 2016, 15, 2217-2228.

(6) Cymer, F.; Beck, H.; Rohde, A.; Reusch, D. Therapeutic monoclonal antibody N-glycosylation - Structure, function and therapeutic potential Biologicals 2018, 52, 1-11.

(7) O'Flaherty, R.; Trbojevic-Akmacic, I.; Greville, G.; Rudd, P. M.; Lauc, G. The sweet spot for biologics: recent advances in characterization of biotherapeutic glycoproteins Expert Rev Proteomics 2018, 15, 13-29.

(8) Miyoshi, E.; Moriwaki, K.; Terao, N.; Tan, C. C.; Terao, M.; Nakagawa, T.; Matsumoto, H.; Shinzaki, S.; Kamada, Y. Fucosylation is a promising target for cancer diagnosis and therapy Biomolecules 2012, 2, 34-45.

(9) Ito, E.; Oka, R.; Ishii, T.; Korekane, H.; Kurimoto, A.; Kizuka, Y.; Kitazume, S.; Ariki, S.; Takahashi, M.; Kuroki, Y.; Kida, K.; Taniguchi, N. Fucosylated surfactant protein-D is a biomarker candidate for the development of chronic obstructive pulmonary disease J Proteomics 2015, 127, 386-394.

(10) Stanley, P.; Taniguchi, N.; Aebi, M. In Essentials of Glycobiology, Varki, A.; Cummings, R. D.; Esko, J. D.; Stanley, P.; Hart, G. W.; Aebi, M.; Darvill, A. G.; Kinoshita, T.; Packer, N. H.; Prestegard, J. H.; Schnaar, R. L.; Seeberger, P. H., Eds.; 2015-2017 by The Consortium of Glycobiology Editors, La Jolla, California: Cold Spring Harbor NY, 2015, pp 99-111.

(11) Stanley, P.; Cummings, R. D. In Essentials of Glycobiology, Varki, A.; Cummings, R. D.; Esko, J. D.; Stanley, P.; Hart, G. W.; Aebi, M.; Darvill, A. G.; Kinoshita, T.; Packer, N. H.; Prestegard, J. H.; Schnaar, R. L.; Seeberger, P. H., Eds.; 2015-2017 by The Consortium of Glycobiology Editors, La Jolla, California: Cold Spring Harbor NY, 2015, pp 161-178.

(12) Harvey, D. J.; Mattu, T. S.; Wormald, M. R.; Royle, L.; Dwek, R. A.; Rudd, P. M. "Internal residue loss": rearrangements occurring during the fragmentation of carbohydrates derivatized at the reducing terminus Anal Chem 2002, 74, 734-740.

(13) Wuhrer, M.; Koeleman, C. A.; Hokke, C. H.; Deelder, A. M. Mass spectrometry of proton adducts of fucosylated $\mathrm{N}$-glycans: fucose transfer between antennae gives rise to misleading fragments Rapid Commun Mass Spectrom 2006, 20, 1747-1754. 
(14) Liu, M. Q.; Zeng, W. F.; Fang, P.; Cao, W. Q.; Liu, C.; Yan, G. Q.; Zhang, Y.; Peng, C.; Wu, J. Q.; Zhang, X. J.; Tu, H. J.; Chi, H.; Sun, R. X.; Cao, Y.; Dong, M. Q.; Jiang, B. Y.; Huang, J. M.; Shen, H. L.; Wong, C. C. L.; He, S. M., Yang, P. Y. pGlyco 2.0 enables precision Nglycoproteomics with comprehensive quality control and one-step mass spectrometry for intact glycopeptide identification Nat Commun 2017, 8, 438.

(15) Nwosu, C.; Yau, H. K.; Becht, S. Assignment of Core versus Antenna Fucosylation Types in Protein N-Glycosylation via Procainamide Labeling and Tandem Mass Spectrometry Anal Chem 2015, 87, 5905-5913.

(16) Tajiri, M.; Kadoya, M.; Wada, Y. Dissociation profile of protonated fucosyl glycopeptides and quantitation of fucosylation levels of glycoproteins by mass spectrometry J Proteome Res 2009, 8, 688-693.

(17) Okada, T.; Sato, Y.; Kobayashi, N.; Sumida, K.; Satomura, S.; Matsuura, S.; Takasaki, M.; Endo, T. Structural characteristics of the N-glycans of two isoforms of prostate-specific antigens purified from human seminal fluid Biochim Biophys Acta 2001, 1525, 149-160.

(18) Turiak, L.; Ozohanics, O.; Marino, F.; Drahos, L.; Vekey, K. Digestion protocol for small protein amounts for nano-HPLC-MS(MS) analysis J Proteomics 2011, 74, 942-947.

(19) Domon, B.; Costello, C. E. A systematic nomenclature for carbohydrate fragmentations in FAB-MS MS spectra of glycoconjugates Glycoconj J 1988, 5, 397-409.

(20) Vekey, K.; Ozohanics, O.; Toth, E.; Jeko, A.; Revesz, A.; Krenyacz, J.; Drahos, L. Fragmentation characteristics of glycopeptides Int J Mass Spectrom 2013, 345, 71-79.

(21) Ozohanics, O.; Turiak, L.; Puerta, A.; Vekey, K.; Drahos, L. High-performance liquid chromatography coupled to mass spectrometry methodology for analyzing site-specific $\mathrm{N}$ glycosylation patterns J Chromatogr A 2012, 1259, 200-212.

(22) Vekey, K.; Pocsfalvi, G. Calculation of the kinetic energy release of charge separation processes Org Mass Spectrom 1992, 27, 1203-1209.

(23) Hinneburg, H.; Stavenhagen, K.; Schweiger-Hufnagel, U.; Pengelley, S.; Jabs, W.; Seeberger, P. H.; Silva, D. V.; Wuhrer, M.; Kolarich, D. The Art of Destruction: Optimizing Collision Energies in Quadrupole-Time of Flight (Q-TOF) Instruments for Glycopeptide-Based Glycoproteomics J Am Soc Mass Spectrom 2016, 27, 507-519.

(24) Yang, H.; Yang, C.; Sun, T. Glycopeptides Characterization using a Stepped HCD Energy Approach on Hybrid Quadrupole Orbitrap Rapid Commun Mass Spectrom 2018, doi: $10.1002 / \mathrm{rcm} .8191$.

(25) Kolli, V.; Dodds, E. D. Energy-resolved collision-induced dissociation pathways of model Nlinked glycopeptides: implications for capturing glycan connectivity and peptide sequence in a single experiment Analyst 2014, 139, 2144-2153.

(26) C Collette, C.; Drahos, L.; De Pauw, E.; Vékey, K. Comparison of the internal energy distributions of ions produced by different electrospray sources Rapid Commun Mass Spectrom 1998, 12, 1673-1678

(27) Nakano, M.; Kakehi, K.; Tsai, M. H.; Lee, Y. C. Detailed structural features of glycan chains derived from alpha1-acid glycoproteins of several different animals: the presence of hypersialylated, O-acetylated sialic acids but not disialyl residues Glycobiology 2004, 14, 431441. 
"For TOC only"

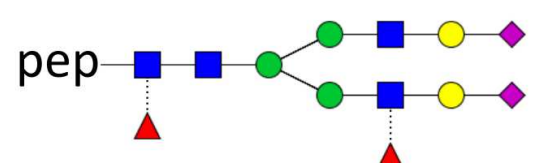

Core fucose ? Antenna fucose

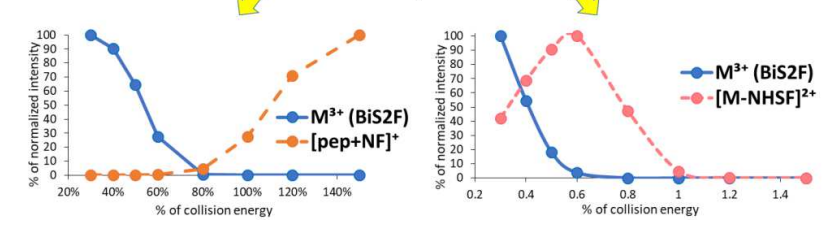

